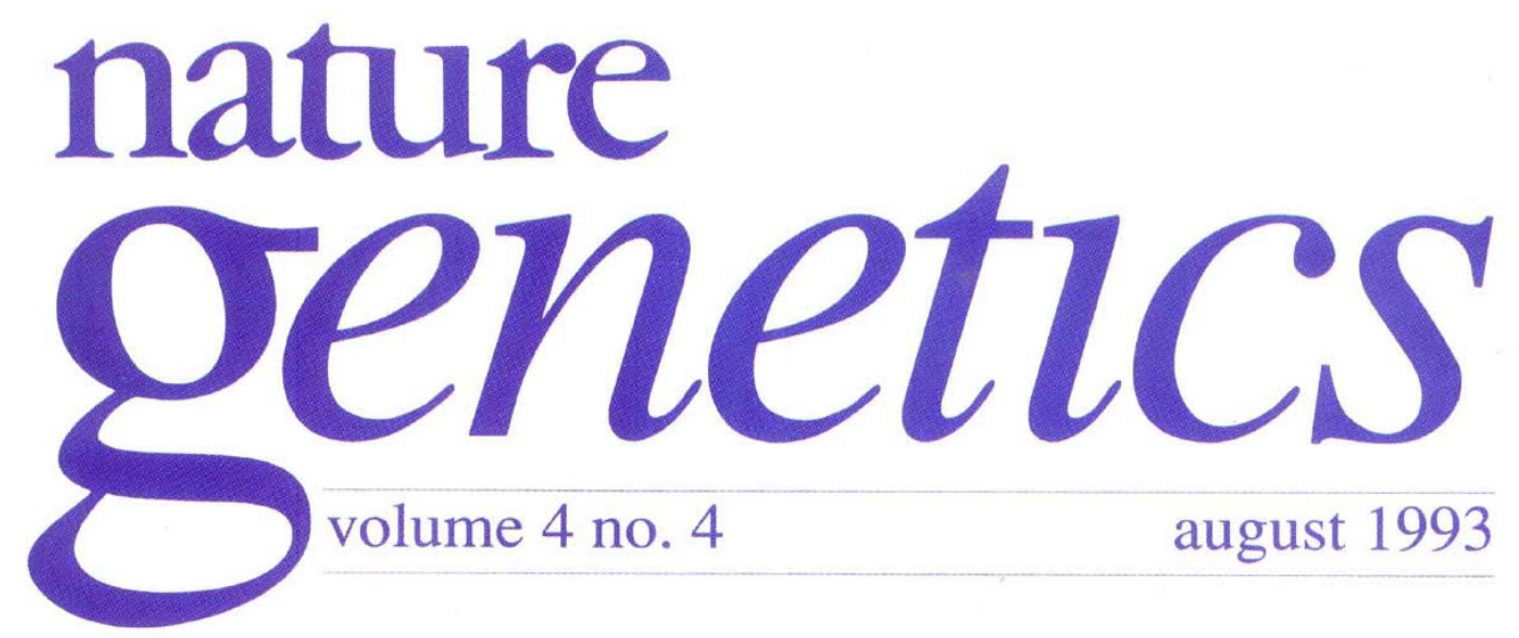

\title{
Collaboration, Canadian style
}

Within scientific research, genetics - and in particular clinical molecular genetics - is as competitive a field as any. With a high level of public awareness feeding off the promise of a new age of predictive medicine, gene therapy and the unravelling of polygenic disorders, there is often a great deal at stake. The highly publicized races to identify the genes responsible for important diseases such as cystic fibrosis, neurofibromatosis and Huntington's disease have resulted in major winners and noticeable losers. Unreasonably, the victors are almost guaranteed future funding with which groups will expand, attracting high calibre scientists and the interest of the community, whereas the losers, having associated themselves with a clearly defined goal, may, for want of a little good luck, find themselves searching for a new focus.

Has this fierce level of competition served the genetics communitywell? Probably not, as evidenced by Canadian geneticists who have come across a system which harnesses the best that the competitive spirit has to offer (a sense of urgency and focused research) without the associated non-productive aspects (limited resources, an anxiety about sharing results and the concomitant isolation).

In the late 1980s, the Canadian government investigated ways of giving the economy a kick start. Its commendable approach was to encourage and promote the transfer of technologies from the research phase to development and production. To do this they created the Canadian Federal Centres of Excellence Network programme. Unlike the networks promoted by the European Science Foundation, the Canadian programme offered substantial research funding to any community that could identify itself as a cohesive group, made up of centres of excellence, in any field of scientific research that had the potential for new industrial partnerships. Canadian geneticists took up the challenge and after conducting a peer-reviewed selection they defined themselves as a group of 23 leading investigators associated with eight universities. This seemingly simple step may, in hindsight, have presented the greatest challenge. The named group of individuals did not include all the genetics research groups in Canada; those considered centres of excellence were included and those that were not, were not. Inevitably the exclusion of some created ill feeling. Nevertheless, the genetics network was launched in 1990 with the laudable aims of promoting collaborative research, instigating industrial partnerships, promoting training and finally to benefit Canadians and their health care system (The genetics diseases network was one of just 15 successful applications from a field of 150 . Applications were peer-reviewed by an international committee and were also assessed for their potential to achieve technological transfer through industrial collaborations).

The network holds annual meetings where the overall direction of the network is monitored and redirected as necessary and a forthright scientific exchange of published and unpublished work takes place in a closed forum and under the provision that no further discussion takes place in public. Does this work in practice? What stops the most competitive groups going along to listen rather than to contribute? Perhaps other aspects of the network have helped to produce a genuine feeling of community so that groups can share their latest findings without fear of losing their competitive edge. These other benefits include the provision of 
centrally-funded core facilities that, at no cost, provide an expert service to any network member. There are twelve core facilities, including relatively standard procedures such as DNA sequencing, oligonucleotide synthesis, in situ mapping and computational analysis, and more advanced facilities such as gene targeting, transgenic modelling, protein sequencing and yeast artificial chromosome production. Each facility is housed in a member's laboratory and funded entirely by the network. If required, work is carried out with no questions asked. Indeed, as Lap-Chee Tsui (the sequencing and YAC production facilities' director) says, the core facilities may even prefer this anonymity to avoid the temptation of intriguing glimpses into the work of the rest of the network. The free movement of (particularly more junior) workers within the network is also centrally funded, encouraging collaboration and training at every opportunity.

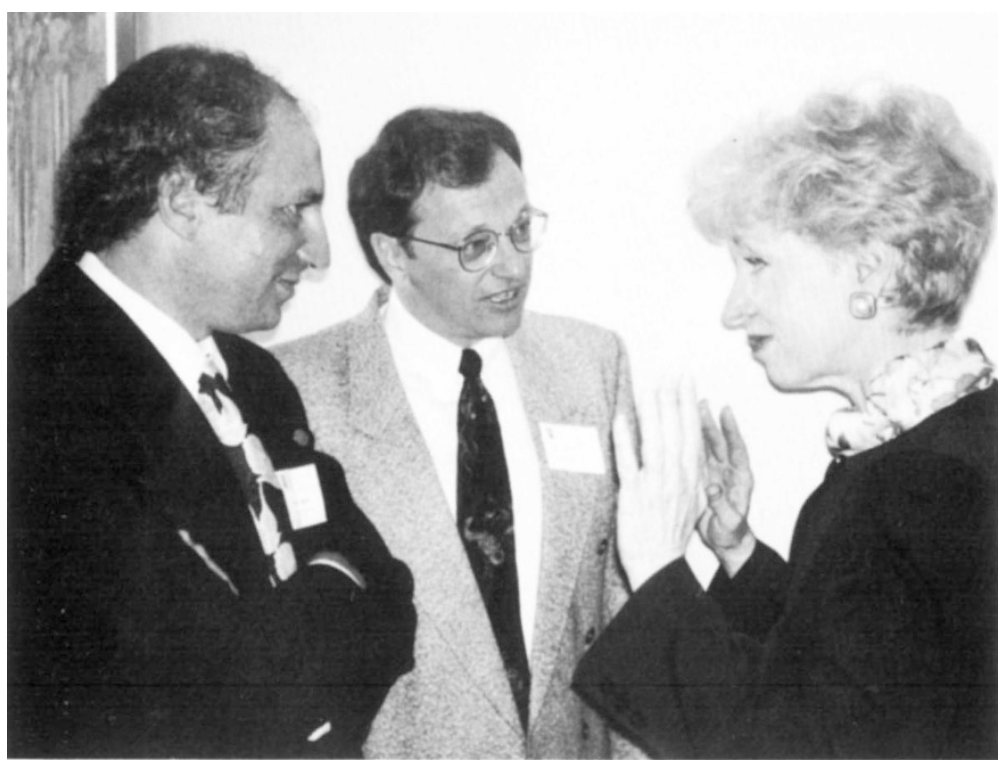

Kim Campbell (newly-elected Canadian prime minister) discusses the Genetics Disease Network with Michael Hayden (left) and Robert Korneluk (centre).

But this seemingly harmonious community is not without its problems. The generous start-up funds (a total of C $\$ 240$ million of which the genetics network received C $\$ 17.5$ million) provided for the first four years, but now the entire initiative has been asked to justify itself in the face of reduced funding. In fact the total allocation for the fifteen networks (including such disparate groups as Space Research, Robotics \& Intelligent Systems and Aging Research) is likely to be halved. Thus, although competition is as fierce as ever, Canadian geneticists have now galvanized themselves into a coordinated effort to compete with, amongst others, the National Centres of Excellence funded High Performance Concrete group.

The founder and recently re-elected director of the genetics network, Michael Hayden (University of British Columbia), is fond of referring to the network as "an institute without walls" in which all members are available to help colleagues with particular problems as well as long term ventures. Although Hayden's group narrowly lost the race to find the Huntington's gene, they have rapidly made up for lost time (see page 398, this issue) thanks in part to the availability of the extensive core facilities and associated personnel that were called upon, at a moments notice, to help Hayden's group complete some of the laborious screening. This unprecedented cooperation and flexibility contributes, says David Shindler, the network managing director, to "a culture change regarding the conduct of research". Part of this culture change involves a new approach to industrial relations in which industry is seen as a partner, with which projects are discussed and shaped at their conception, rather than a marketing tool to be wheeled in at the end of a project.

Hayden quotes from a modest but nonetheless impressive list of network achievements and points out that the network has so far attracted more than C\$15 million in industrial support (mainly from Merck Frosst) with more on the horizon. High on this list is the discovery of the gene for myotonic dystrophy and recent advances in gene targeting protocols. Such discoveries might well have come about in the absence of the network, but the researchers who led these groups (Bob Korneluk of the Children's Hospital of Eastern Ontario and Jamey Marth of UBC respectively) acknowledge important contributions from the network. Although Korneluk also received generous funding from the network, he believes that "the noncompetitive collaboration, which may simply be talking to well respected geneticists, was a significant factor". Korneluk goes on to say "but the real advantage [of the network] comes now, with the establishment of the core facilities" and it is hard to argue with this. In the light of the success the network has achieved in its first years, it might well represent one of the greatest lost opportunities if the Canadian government sticks to its plan to cut drastically funding to the project. One can only hope that the new Prime Minister, Kim Campbell, will take a closer look at what can be achieved through collaborative research, community spirit and Canadian style. 